\title{
Corrigendum
}

\section{Karyological analysis of bitter gourd (Momordica charantia L., Cucurbitaceae) from Southeast Asian countries - Corrigendum}

Nadia Kausar, Zubaida Yousaf, Afifa Younas, Hafiza Sadia Ahmed, Madiha Rashid, Ayesha Arif and Hafiza Ayesha Rehman

DOI: https://doi.org/10.1017/S147926211400077X Published online by Cambridge University Press: 9 July 2014

When originally published Madiha Rashid's name was spelt incorrectly. This has now been corrected in the online version of the article.

\section{Reference}

Kausar, N., Yousaf, Z., Younas, A., Ahmed, H., Rashid, M., Arif, A., \& Rehman, H. (2015). Karyological analysis of bitter gourd (Momordica charantia L., Cucurbitaceae) from Southeast Asian countries. Plant Genetic Resources, 13(2), 180-182. doi:10.1017/ S147926211400077X 